\title{
Fast Aberration Measurement in Multi-Dimensional STEM
}

\author{
Andrew R. Lupini ${ }^{1,3}$, Miaofang Chi $^{2}$, Sergei V. Kalinin ${ }^{2,3}$, Albina Y. Borisevich ${ }^{1,3}$, Juan Carlos Idrobo ${ }^{2}$, \\ Stephen Jesse 2,3 . \\ ${ }^{1}$ Materials Science \& Technology Division, Oak Ridge National Laboratory, Oak Ridge, TN \\ ${ }^{2}$ Center for Nanophase Materials Sciences, Oak Ridge National Laboratory, Oak Ridge, TN \\ ${ }^{3}$ Institute for Functional Imaging of Materials, Oak Ridge National Laboratory, Oak Ridge, TN
}

A general goal of electron microscopy could perhaps be stated as the desire to extract as much information about a sample as possible. However, fundamental restrictions arise from limited resolution, sample damage, and the lack of phase sensitivity. Multi-dimensional (scanning) transmission electron microscopy, (S)TEM, provides effective routes to attack each of these problems $[1,2,3]$.

The main resolution limits in high-performance (S)TEM are due to the imperfections or 'aberrations' of the electron lenses. Correction of these aberrations requires the ability to quickly and accurately measure lens aberrations to align or 'tune' an aberration-corrector. The conventional method to measure aberrations in the TEM is to record a series of bright field (BF) images in a Zemlin tableau. However, the reciprocity-equivalent method in STEM of recording a series of tilted BF images is inherently inefficient if only a single BF detector is available. Aberration measurement methods based on recording and analyzing the whole scattering distribution (Ronchigram) provide an efficient use of the signal available in the STEM, however, normally only a small number of probe positions is used [3, 4]. We have recently demonstrated a method based on acquiring Ronchigrams at a multiplicity of probe positions [5]. This procedure results in 4-dimensional dataset, which can be transformed to give an array of real-space images of a sample. Cross-correlating the resulting images gives the gradient of the aberration function, from which the aberrations are determined by a least-squares fit [5]. This procedure provides rapid, accurate aberration measurements and can in principle be extended to arbitrary-order measurements. Figure 1 shows an example aberration measurement based on this method. A series of Ronchigrams was recorded with a nominal $1 \mathrm{~nm}$ step between probe positions. A typical Ronchigram is shown, along with measured and fitted shifts, which are used to find the aberration function.

Figure 2 shows several different BF images generated from the example data set. The $64 \times 64$ probe positions yield a 64x64 element image, as might be expected. Because the defocus was measured as approximately $-250 \mathrm{~nm}$ the high-angle annular dark field (HAADF) image becomes rather blurred, whereas a single-pixel BF image appears sharp but noisy (a). By summing several pixels to produce a larger BF detector, the resulting BF image becomes more blurred (delocalized) (b). However, we can consider the $\mathrm{CCD}$ as being equivalent to an array of BF detectors, each producing an image from a slightly different angle. In the approximation used here, this angular change means that the images from subsequent pixels in the binned dataset are shifted by roughly $0.25 \mathrm{~nm}$, i.e. by about $4 \mathrm{x}$ finer than the nominal distance between probe positions. Thus the same dataset of $64 \times 64$ probe positions can be used to construct an image with about $4 \mathrm{x}$ higher spatial sampling in each dimension and a better signal to noise ratio than from a single pixel detector (c). [6] 
References:

[1] O.L. Krivanek, N. Dellby \& A.R. Lupini, Ultramicroscopy 78 (1999) 1-11.

[2] T.J. Pennycook, et al, Ultramicroscopy, Ultramicroscopy 151 (2015) 160-167.

[3] J. M. Rodenburg, B. C. McCallum and P. D. Nellist, Ultramicroscopy, 48 (1993), p. 304.

[4] H. Sawada, et al., Ultramicroscopy 108(11), 1467-75.

[5] A.R. Lupini, M. Chi \& S. Jesse, Journal of Microscopy (2016) DOI: 10.1111/jmi.12372.

[6] Research supported by Division of Materials Sciences and Engineering Division, Office of Basic Energy Sciences, U.S. DOE (ARL, AYB) and by Oak Ridge National Laboratory's Center for Nanophase Materials Sciences (CNMS), which is sponsored by the Scientific User Facilities Division, Office of Basic Energy Sciences, U.S. Department of Energy (MC, SVK, JCI, SJ).

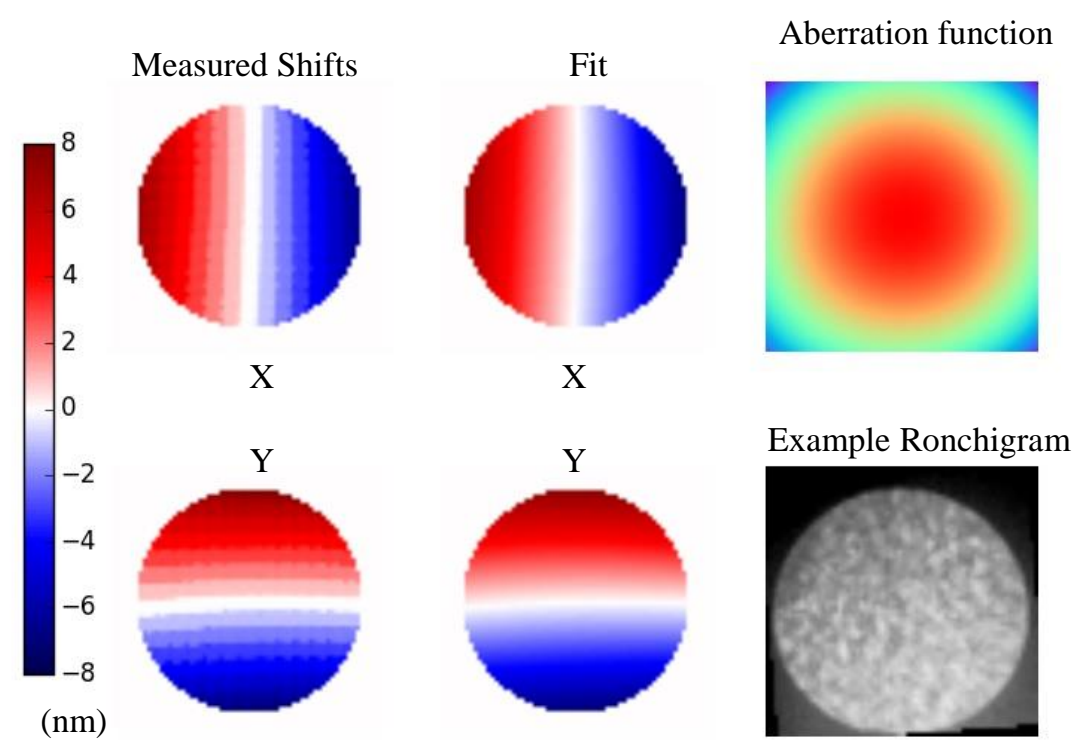

Figure 1. Example measurement of aberrations from a Nion UltraSTEM ${ }^{\mathrm{TM}}$ 200. Measured defocus is approximately $-250 \mathrm{~nm}$. Figure adapted from reference [5].
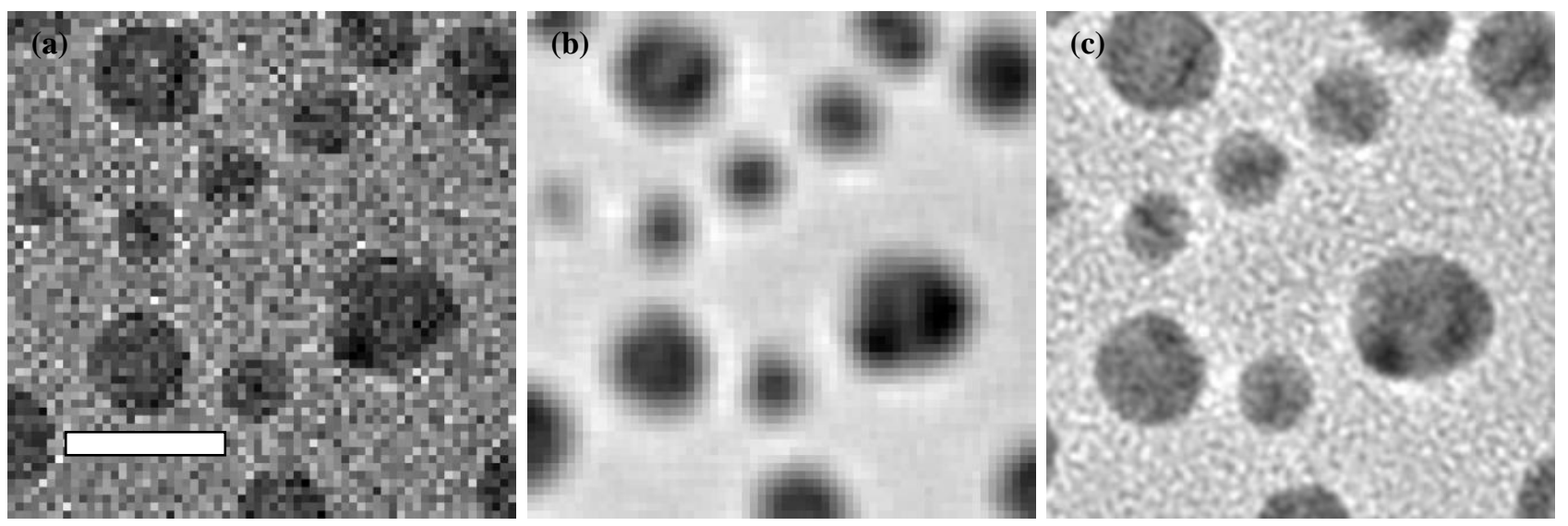

Figure 2. (a) BF image generated from a single pixel from the Ronchigram series. Scale bar is nominally $20 \mathrm{~nm}$. (b) As more pixels are used to form a BF image, the noise improves but fine details are lost. (c) By undoing the induced shifts, the same Ronchigram dataset is used to generate an image with spatial sampling finer than the probe spacing. 